\title{
Results of CHOP chemotherapy for diffuse large B-cell lymphoma
}

A.E. Hallack Neto,

J. Pereira, B. Beitler, D.A.F. Chamone, P.D. Llacer, F.L. Dulley, M.C.M.A. Macedo and A. Chaoubah
Departamento de Hematologia, Hospital das Clínicas, Universidade de São Paulo, São Paulo, SP, Brasil
Correspondence

A.E. Hallack Neto

Avenida Rio Branco, 2679

Sala 714

Centro

36010-012 Juiz de Fora, MG

Brasil

Fax: +55-32-3215-1523

E-mail: abrahallack@ig.com.br

Publication supported by FAPESP.

$\ldots \ldots \ldots \ldots \ldots \ldots \ldots$

Received September 22, 2004

Accepted June 12, 2006

\begin{abstract}
Patients with diffuse large B-cell lymphoma treated in a University Hospital were studied from 1990 to 2001. Two treatment regimens were used: ProMACE-CytaBOM and then, from November 1996 on, the CHOP regimen. Complete remission (CR), disease-free survival (DFS), and overall survival (OS) rates were determined. Primary refractory patients and relapsed patients were also assessed. A total of 111 patients under 60 years of age were assessed and ranked according to the international prognostic index adjusted to age. Twenty (18\%) of them were classified as low risk, $40(36 \%)$ as intermediate risk, 33 $(29.7 \%)$ as high intermediate risk, and $18(16.3 \%)$ as high risk. Over a five-year period, OS and DFS rates were 71 and 59\%, respectively, for all patients. For the same time period, OS and DFS rates were 72.8 and $61.3 \%$, respectively, for 77 patients treated with CHOP chemotherapy and 71.3 and $60 \%$ for patients treated with the ProMACECytaBOM protocol. There was no significant difference in OS or DFS between the two groups. Eleven of 50 refractory and relapsed patients were consolidated with high doses of chemotherapy. Three received allogenic and 8 autologous bone marrow transplantation. For the latter, CR was $62.5 \%$ and mean OS was 41.1 months. The clinical behavior, CR, DFS, and OS of the present patients were similar to those reported in the literature. We conclude that both the CHOP and ProMACE-CytaBOM protocols can be used to treat diffuse large Bcell lymphoma patients, although the CHOP protocol is preferable because of its lower cost and lower toxicity.
\end{abstract}

\section{Introduction}

Non-Hodgkin's lymphomas comprise a heterogeneous group of lymphoid tissue cancer with different histological subtypes and clinical features. They represent the fifth most common type of cancer in Brazil, with
Key words

- Diffuse large B-cell lymphoma

- CHOP lymphoma treatment

- ProMACE-CytaBOM

treatment 
Western world, the diffuse large B-cell lymphoma (DLBCL) subtype is the most common one (4).

Treatment requires assessing the patient's functions and prognostic factors. Curative treatment for these patients is based on an anthracyclic antibiotic-containing chemotherapy course (5). Overall survival rates for patients who scored high on the age-adjusted international prognostic index (aIPI) were lower than $30 \%$ with conventional treatments. The best therapeutic approach to these patients has not been established (6).

Based on the hypothesis raised by Goldie and Coldman (7), according to which an increase in the number and/or intensity of chemotherapy doses would elicit a better response, several intensive protocols have been designed and tested. However, none of them proved to be superior to the CHOP regimen, which is still the first choice for the treatment of DLBCL patients, apart from experimental protocols (8).

In the group of primary refractory and relapsed patients previously treated by salvage chemotherapy, 20 to $40 \%$ reached complete remission. Nonetheless, if autologous bone marrow transplantation is not consolidated, overall 5-year survival will be lower than $10 \%$. If consolidation is used, overall survival will increase to $46 \%$ (9).

In Brazil, studies are needed in order to find out if the patients' clinical and biological behavior is equivalent to that described in other geographical areas. If this proves not to be the case, there should be an adaptation to these new conditions. In the present study, we assessed complete remission, disease-free survival, and overall survival rates for DLBCL patients under 60 years of age who were treated at the Hematology Department of the Hospital das Clínicas, University of São Paulo, over a period of 12 years. The refractory and relapsed patients were also assessed regarding the type of salvage chemotherapy used and the corresponding responses.

\section{Patients and Methods}

Between January 1990 and October 1996, the DLBCL patients were treated with ProMACE-CytaBOM $\left(60 \mathrm{mg} / \mathrm{m}^{2}\right.$ prednisona, $25 \mathrm{mg} / \mathrm{m}^{2}$ doxurubicin, $650 \mathrm{mg} / \mathrm{m}^{2}$ cyclophosphamide, $120 \mathrm{mg} / \mathrm{m}^{2}$ etoposide, $300 \mathrm{mg} /$ $\mathrm{m}^{2}$ cytarabina, 5 units $/ \mathrm{m}^{2}$ bleomycin, $1.4 \mathrm{mg} /$ $\mathrm{m}^{2}$ vincristine, and $120 \mathrm{mg} / \mathrm{m}^{2}$ methotrexate) chemotherapy. From November 1996 to December 2001 they underwent the CHOP $\left(750 \mathrm{mg} / \mathrm{m}^{2}\right.$ cyclophosphamide, $50 \mathrm{mg} / \mathrm{m}^{2}$ doxorubicin (Adriamycin), $1.4 \mathrm{mg} / \mathrm{m}^{2}$ vincristine, and $500 \mathrm{mg}$ prednisone) regimen. The histology of the patients was reviewed for inclusion in the present analysis.

The aIPI was determined for all patients $(\mathrm{N}=111)$, who were divided into four groups: low $(\mathrm{N}=20)$, low intermediate $(\mathrm{N}=40)$, high intermediate $(\mathrm{N}=33)$, and high risk $(\mathrm{N}$ $=18$ ). In order to compare complete remission, overall survival, and disease-free survival rates according to the aIPI among the four groups, the groups were rearranged into two groups, mainly due to the small number in each. Low and low intermediate aIPI patients were assigned to the low risk-adapted group $(\mathrm{N}=60)$ and high intermediate and high aIPI patients were assigned to the high risk-adapted group $(\mathrm{N}=51)$.

Patients were submitted to 4 to 8 treatment cycles according to their clinical classification. Patients in stage I or II or in the bulky stage (tumor size over $7 \mathrm{~cm}$ or cardiothoracic index over 1/3) who achieved complete remission also received a course of involved field radiation (3600 cCy). Patients who presented lymphoma in their facial bones or other bones, in the testes, and Waldeyer's ring also underwent radiation. Patients with lymphoma in their testes, ovaries, and paravertebral region received 4 intrathecal cycles of $12 \mathrm{mg}$ methotrexate and $2 \mathrm{mg}$ dexamethasone as prophylaxis against relapse in the central nervous system.

The refractory and relapsed patients received different salvage chemotherapy treat- 
ments. Eight of them were consolidated with autologous bone marrow transplantation and three with allogenic transplantation.

Confirmed complete remission was defined by the lack of all lymphoma-related signs and symptoms over a period of at least 4 weeks. The criteria for partial remission were a lesion reduction of $50 \%$ or over by adding up the results of the two major perpendicular values of the measurable lesions, lack of development of pre-existing lesions, appearance of new lesions, and clinical deterioration. Complete mass reduction and persistence of bone marrow infiltration were also considered to be partial remission. Any response lower than partial remission was classified as refractory disease. Development of residual lesions of $25 \%$ or more and the appearance of new lesions implied relapse (10).

All patients underwent a diagnostic workup consisting of tumor biopsy; history-taking; biometric tests; complete blood count; liver and kidney evaluation; serum lactate dehydrogenase (LDH) and $\beta-2$ microglobulin measurements; serum protein electrophoresis; HBV, HCV, HIV, HTLV I and II, syphilis, and South American trypanosomiasis serologies; electrocardiogram; echocardiogram; chest radiography; bilateral bone marrow biopsy; lumbar puncture, and neck, chest, abdomen, and pelvis computed tomography. Patients whose Waldeyer's ring had been affected underwent endoscopy and those whose facial bones were affected underwent brain and face computed tomography. The Ann Arbor criteria were used for re-classification (11), first after the fourth cycle and then at the end of treatment. The criteria were then applied every 3 months during the first 2 years and every 6 months thereafter.

The Student $t$-test, Fisher exact test, and chi-square test, where appropriate, were used for statistical analysis of the data. Overall survival and disease-free survival rates were analyzed by the Kaplan-Meyer method. The survival curves for the two groups were compared by the log-rank test.

Overall survival was determined considering the period from the beginning of treatment to patient death, loss to follow-up, or last assessment. Disease-free survival rates were only analyzed for patients with complete remission considering the period from remission to progression, relapse, or death from any cause. The patients were censored on the day of last observation, or transplantation. For multiple analysis, the Cox regression model was applied to the variables that showed an impact on overall survival curves in univariate analysis. Data were analyzed using the Statistical Software Package for the Social Sciences (SPSS version 10.0 for Windows; SPSS Inc., Chicago, IL, USA).

\section{Results}

Of the 111 patients studied, 77 received the CHOP regimen and 34 the ProMACECytaBOM regimen. We only studied patients under 60 years of age (mean: 38.7 years) in order to avoid interference by agerelated limitations. Accordingly, lower intensity-dosed regimens were used for the population over 60 years old.

The two groups were similar, except for a larger number of high LDH cases in the CHOP group (Table 1). Eighty-one (73\%) of 111 patients obtained complete remission (95\% CI: 64.7; 81.3), with overall survival of more than 60 months (mean: 88 months) (95\% CI: 78.4; 97.8), and a disease-free survival rate of $59 \%$ over 60 months (mean: 93.3 months; 95\% CI: 73.9; 112.8).

The complete remission rate for patients ranked I or II was $83.1 \%$, overall survival rate over 60 months was $84.2 \%$ (mean: 101.3 months; 95\% CI: 90.8; 111.8), and diseasefree survival rate over 60 months was $74.8 \%$ (mean: 74.7 months; 95\% CI: 62.6; 86.8). Complete remission rate for the 52 patients staged III or IV was $61.5 \%$, and overall survival and disease-free survival rates over 
60 months were 55.1 and $37.1 \%$, respectively.

Mean overall survival was 73.4 months (95\% CI: 57.7; 89.1), and mean disease-free survival was 65.7 months (95\% CI: 39.57 ; 91.88; Figure 1A and B). The complete remission, overall survival, and disease-free survival rates for patients staged I or II were significantly higher than those for patients in stages III and IV ( $\mathrm{P}=0.011,0.0124$, and 0.0004).

Sixty patients were ranked as low riskadapted, 20 at low risk and 40 at low intermediate risk. Fifty-one patients were ranked as high risk-adapted, 33 at high intermediate risk and 18 at high risk (Table 1). In the low risk-adapted group, complete remission rate was $78.3 \%$, overall survival rate over 60 months was $84.3 \%$ (mean: 101.87 months; 95\% CI: $91.7 ; 112.1$ ), and disease-free survival rate over 60 months was $68.12 \%$ (mean:

Table 1. Characteristics of patients with diffuse large B-cell lymphoma treated with CHOP or ProMACE-CytaBOM.

\begin{tabular}{lccc}
\hline & CHOP & ProMACE-CytaBOM & Total \\
\hline $\begin{array}{l}\text { Age (years) } \\
\text { Sex }\end{array}$ & $39.87 \pm 12.31$ & $36.06 \pm 12.03$ & - \\
F & $35(45.5 \%)$ & $18(52.9 \%)$ & $53(47.7 \%)$ \\
M & $42(54.5 \%)$ & $16(47.1 \%)$ & $58(52.3 \%)$ \\
Total number of patients & $77(100 \%)$ & $34(100 \%)$ & $111(100 \%)$ \\
B symptoms & $46(59.7 \%)$ & $25(73.5 \%)$ & $71(64 \%)$ \\
Extranodal & $31(40.3 \%)$ & $17(50 \%)$ & $48(43.2 \%)$ \\
BM+ & $11(14.3 \%)$ & $3(8.8 \%)$ & $14(12.6 \%)$ \\
Bulky & $44(57.1 \%)$ & $17(50 \%)$ & $61(55 \%)$ \\
High LDH & $52(67.5 \%)$ & $15(44.1 \%)^{\star}$ & $67(60.4 \%)$ \\
Ann Arbor stage & $41(53.2 \%)$ & $18(52.9 \%)$ & $59(53.2 \%)$ \\
$\quad$ I and II & $36(46.8 \%)$ & $16(47.1 \%)$ & $52(46.8 \%)$ \\
$\quad$ III and IV & & & $60(54.1 \%)$ \\
alPI & $41(53.2 \%)$ & $19(55.9 \%)$ & $51(45.9 \%)$ \\
$\quad$ Low & $36(46.8 \%)$ & $15(44.1 \%)$ & \\
High & & & \\
\hline
\end{tabular}

Data are reported as means \pm SD or as number of patients and percent in parentheses. $\mathrm{CHOP}=750 \mathrm{mg} / \mathrm{m}^{2}$ cyclophosphamide, $50 \mathrm{mg} / \mathrm{m}^{2}$ doxorubicin (Adriamycin), 1.4 $\mathrm{mg} / \mathrm{m}^{2}$ vincristine, and $500 \mathrm{mg}$ prednisone; ProMACE-CytaBOM $=60 \mathrm{mg} / \mathrm{m}^{2}$ prednisone, $25 \mathrm{mg} / \mathrm{m}^{2}$ doxurubicin, $650 \mathrm{mg} / \mathrm{m}^{2}$ cyclophosphamide, $120 \mathrm{mg} / \mathrm{m}^{2}$ etoposide, $300 \mathrm{mg} / \mathrm{m}^{2}$ cytarabina, 5 units $/ \mathrm{m}^{2}$ bleomycin, $1.4 \mathrm{mg} / \mathrm{m}^{2}$ vincristine, $120 \mathrm{mg} / \mathrm{m}^{2}$ methotrexate; $\mathrm{B}$ symptoms $=$ fever $>38^{\circ} \mathrm{C}$, night sweats, weight loss $>10 \%$; Extranodal $=$ involvement of one or more distant extranodal organs; $\mathrm{BM}+=$ bone marrow involvement; Bulky = mass $>7 \mathrm{~cm}$ in diameter; $\mathrm{LDH}=$ lactate dehydrogenase; alPI = ageadjusted international prognostic index.

${ }^{*} \mathrm{P}=0.020$ compared to CHOP (chi-square test).
69.6 months; $95 \%$ CI: $56.7 ; 82.5)$. In the high risk-adapted group, complete remission rate was $66.7 \%$, overall survival rate was $54.8 \%$ over 60 months (mean: 67.8 months; 95\% CI: 53.1; 82.5), and diseasefree survival rate over 60 months was $46.6 \%$ (mean: 79.6 months; 95\% CI: 53.5; 105.7), with statistical significance for overall survival and disease-free survival $(\mathrm{P}=0.002$ and 0.02).

Overall survival over 60 months for patients who achieved complete remission was 79.7\% (mean: 97.6 months; 95\% CI: 87.9; 107.4), and was higher than the one for refractory patients $(\mathrm{P}<0.0001)$. Twentyfour $(22 \%)$ of 111 patients were primary refractory patients and 26 (23\%) were early relapsed patients. Twenty-nine of these $(58 \%)$ presented high intermediate to high risk levels.

When multivariate analysis was performed, the factors that influenced overall survival were aIPI, which was found to be the independent predicting factor $(\mathrm{P}=0.009)$ with a relative risk of 3.2 (95\% CI: 1.33; $7.75)$, and primary therapeutic response $(\mathrm{P}<$ $0.0001)$ with a relative risk of $4.61(95 \% \mathrm{CI}$ : 2.07; 10.3).

There was no statistically significant difference in the complete remission rates between patients treated with the CHOP (68.8\%) or the ProMACE-CytaBOM (82.4\%; $\mathrm{P}=0.139$ ) regimens. Overall survival over 60 months was $72.8 \%$ in the CHOP group (mean: 65.8 months; 95\% CI: 57.8; 73.7) and $71 \%$ (mean: 89 months) in the ProMACE-CytaBOM group (95\% CI: 79.4; 122.1; $\mathrm{P}=0.85$; Figure $1 \mathrm{C})$.

Also, there was no statistically significant difference in the disease-free survival rates between the $\mathrm{CHOP}$ group and the ProMACE-CytaBOM group. Disease-free survival over 60 months was $61.3 \%$ for patients treated with the CHOP protocol (mean: 101 months; 95\% CI: 79.7; 122.1) and 60\% (mean: 66.6 months) for patients treated with ProMACE-CytaBOM chemotherapy (95\% 

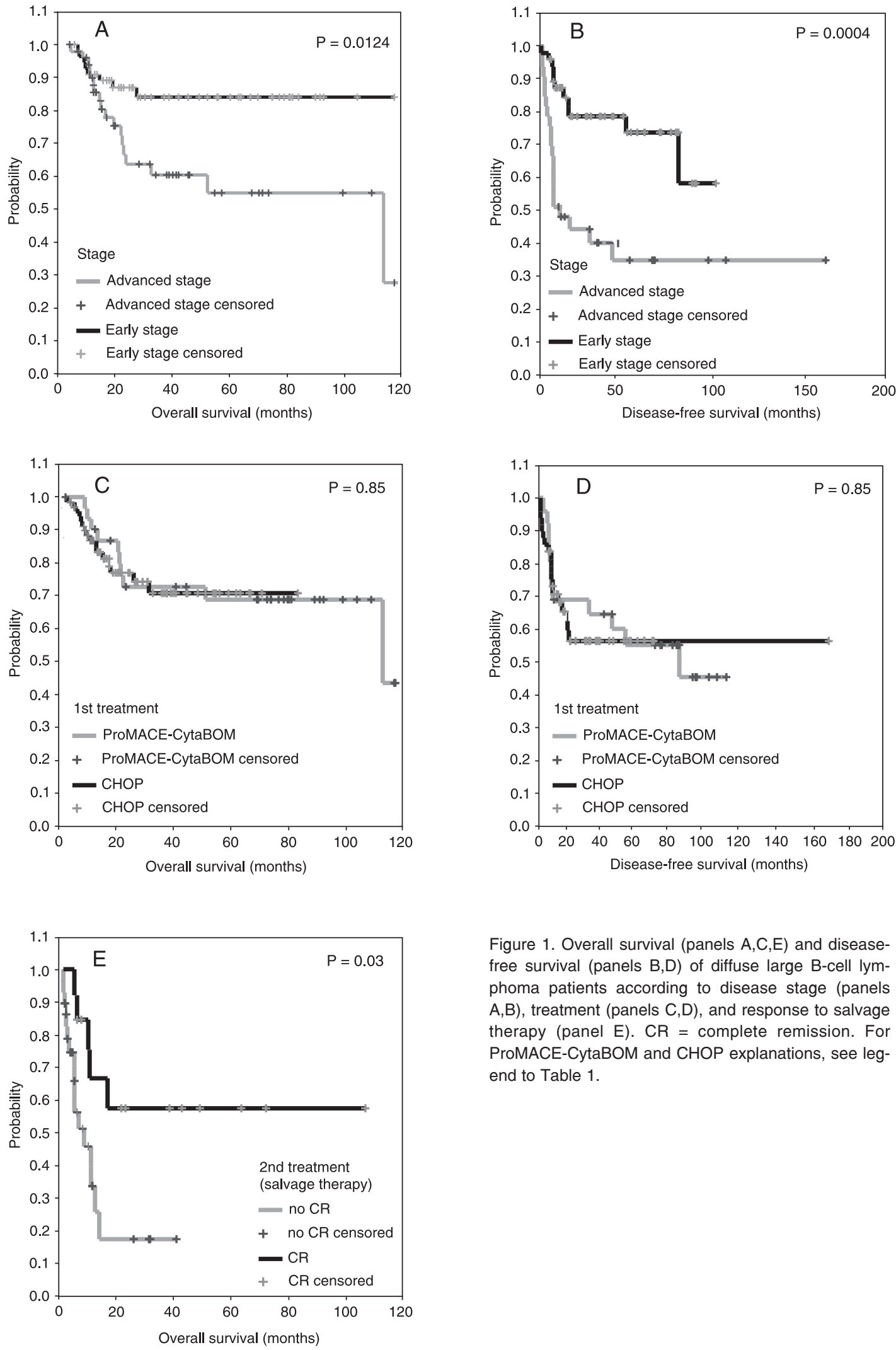

Figure 1. Overall survival (panels $A, C, E)$ and diseasefree survival (panels B,D) of diffuse large B-cell lymphoma patients according to disease stage (panels $A, B$ ), treatment (panels $C, D$ ), and response to salvage therapy (panel E). CR = complete remission. For ProMACE-CytaBOM and CHOP explanations, see legend to Table 1. 
CI: $50.4 ; 82.7 ; \mathrm{P}=0.85$; Figure 1D).

For the patients who received salvage chemotherapy without bone marrow transplantation, the complete remission rate was $23.7 \%$. However, the patients who responded to the salvage chemotherapy and those who were chemoresistant and received bone marrow transplantation both presented a complete remission rate of $54.5 \%$ and overall survival rate over 60 months of $22.7 \%$ (mean: 31.3 months; 95\% CI: 6.1; 56.5). Complete remission occurred in $62.5 \%$ of patients who received autologous bone marrow transplantation, with an overall survival rate of $33.3 \%$ over 60 months (mean: 41.1 months; $95 \%$ CI: 7.8; 74.5).

We observed a trend towards higher levels of complete remission in patients submitted to consolidation with bone marrow transplantation compared to the group that only received salvage chemotherapy. The overall survival rate over 40 months was $63 \%$ for the patients who achieved complete remission (mean: 69.5 months) (95\% CI: 44.9; 94.0) and $28.1 \%$ (mean: of 16.2 months) for the patients who did not reach complete remission (95\% CI: 9.6; 22.9; $\mathrm{P}=0.03$; Figure 1E).

\section{Discussion}

Biological breakthroughs in our knowledge of malignancies have opened new therapeutic perspectives in oncology. This fact, coupled with the emotional burden associated with the diagnosis of a malignant disease, can lead to the replacement of traditional treatments with other options not yet validated, often involving greater toxicity and costs, in spite of the lack of any demonstrable efficacy. Therapeutic decisions should be increasingly based on clinical studies whose populations have features that mimic as closely as possible those presented by the patient in question. However, treatment of malignancies in developing countries is based on studies of populations whose socioeco- nomic features are different from those of populations from developed countries (12).

Based on Goldie and Coldman's (7) hypothesis, several researchers started using more aggressive protocols. Starting in 1980, several drug combinations appeared, with the addition of other drugs like VePesid (VP-16) and methotrexate, or with increased doses and/or reduced cycle intervals. The separate evaluation of these regimens, especially third-generation ones (MACOP-B, ProMACE-CytaBOM, COP-BLAM IV, LNH-84), has proved their superiority over the CHOP protocol $(13,14)$. However, a randomized multi-center study comparing the ProMACE-CytaBOM (prednisone, cyclophosphamide, doxorubicin hydrochloride, etoposide, cytarabine, bleomycin, vincristine, and methotrexate), the MACOP-B, and the m-BACOD regimens with the CHOP did not show any statistically significant difference regarding complete remission, overall survival, and disease-free survival. There was a trend towards higher toxicity in the groups treated with second- or third-generation regimens (8). Later, a study comparing the CHOP and the ProMACE-CytaBOM did not demonstrate any benefits in using the latter (14). Therefore, the CHOP protocol is still the first choice for the treatment of DLBCL patients.

In our cases, as well as in the literature, we did not observe any statistically significant difference in complete remission, overall survival, or disease-free survival rates between the CHOP and the ProMACECytaBOM regimens $(\mathrm{P}=0.139)(8,14)$. However, there was still a trend towards higher complete remission rates in the group receiving the ProMACE-CytaBOM protocol, probably explained by the larger number of high LDH patients in the group treated with CHOP. However, these results should be analyzed with caution since ours was a nonrandomized study with a limited number of patients treated over a long period of time. All the ProMACE-CytaBOM patients were 
treated in the early years (1990-1996) and supportive care might have changed over subsequent years.

Unlike the data in the study that defined IPI, more than $50 \%$ of our cases showed early stages at diagnosis, probably due to the exclusion of patients over 60 years of age. This contributed to a larger portion of patients at low aIPI risk and their complete remission, overall survival and disease-free survival rates.

As there was no difference in overall survival and disease-free survival among the patients with low intermediate or low aIPI risk and among the high intermediate and high aIPI risk, it was possible to rearrange the patients into a low risk-adapted group and a high risk-adapted group (15). The patients in the low risk-adapted group presented statistically higher overall survival and disease-free survival rates than those in the high risk-adapted group. The variable with a major impact on overall survival was complete remission after the first treatment $(\mathrm{P}<0.0001)$. Forty-seven $(58 \%)$ of the 81 patients who achieved complete remission belonged to the low risk-adapted group and these factors were independently predictive of overall survival in multivariate analysis.

The patients with unfavorable aIPI, advanced stage of disease (stages III and IV), or tumor size of more than $10 \mathrm{~cm}$ ("bulky") achieve about $60 \%$ complete remission, although 50\% relapse, and only one third of the patients with disseminated disease are cured $(16,17)$. These results are similar to those reported here since the high risk-adapted complete remission rate was $66.7 \%$, overall survival rate was $54.8 \%$ (mean of 67.8 months; 95\% CI: 53.1; 82.5) and diseasefree survival rate over 60 months was $46.6 \%$ (mean: 79.6 months; 95\% CI: 53.5; 105.7), with statistical significance for overall survival and disease-free survival.

We believe that the use of a protocol different from the CHOP to treat DLBCL patients is only justified for patients in ex- perimental protocols. The CHOP protocol has efficacy similar to that of third-generation protocols for the treatment of DLBCL patients and is less toxic, requires a shorter hospital stay and, therefore, is of lower cost. Recent results have shown benefits in the association of the CHOP protocol and antiCD20 monoclonal antibodies (rituximab) $(18,19)$, but the use of these drugs makes the treatment of DLBCL patients rather more expensive, with the public health system of developing countries having difficulty in implementing such protocols.

Of the patients refractory to chemotherapy and patients with early relapse, only 20 to $40 \%$ achieve complete remission with salvage chemotherapy and less than $10 \%$ will be alive in 5 years (20-22). Survival rates rise to $46 \%$ when the patients who respond to salvage chemotherapy are consolidated with transplantation (23).

In the cases reported here, refractory and relapsed patients were either given different salvage chemotherapy followed or not by consolidation with bone marrow transplantation. According to the literature, the complete remission rate for patients who did not undergo a transplantation was $23.7 \%$ and overall survival rate was $8 \%$ over 5 years $(21,22)$. Nonetheless, in the present study, the patients consolidated with bone marrow transplantation achieved a complete remission rate of $54.5 \%$, with an overall survival rate of $22.7 \%$ over 5 years and a mean survival of 31.3 months. By excluding those receiving allogenic transplantation, the complete remission rate rose to $62.5 \%$ and mean survival was 41.1 months. A recent study has demonstrated that the overall survival rate was $34 \%$ over 4 years (24). Similarly, in our series this rate was $33.3 \%$.

Both the CHOP and the ProMACECytaBOM protocols can be used to treat our DLBCL patients. Nevertheless, due to lower costs and toxicity, we should use the CHOP protocol. Because of their low-survival rate, patients with high risk for aIPI should be 
approached in a different way in experimental regimens, such as consolidation with au- tologous bone marrow transplantation in their first complete remission.

\section{References}

1. Instituto Nacional do Câncer. Linfoma não Hodgkin. http://www.inca. gov.br. Accessed June 29, 2004.

2. Mirra AP, Latorre MRDO, Veneziano DB. Incidência de câncer no município de São Paulo, Brasil 1997-1998. Mortalidade de câncer no município de São Paulo, Brasil. Tendência no período de 19691998. Brasília: Ministério da Saúde; 2001.

3. Rabkin CS, Ward MH, Manns A, Blattner WA. Epidemiology of nonHodgkin's lymphomas. In: Magrath IT (Editor), The non-Hodgkin's lymphomas. London: Arnold; 1997. p 171-186.

4. Shih LY, Liang DC. Non-Hodgkin's lymphomas in Asia. Hematol Oncol Clin North Am 1991; 5: 983-1001.

5. McKelvey EM, Gottlieb JA, Wilson HE, Haut A, Talley RW, Stephens $R$, et al. Hydroxyldaunomycin (Adriamycin) combination chemotherapy in malignant lymphoma. Cancer 1976; 38: 1484-1493.

6. Kimby E, Brandt L, Nygren P, Glimelius B. A systematic overview of chemotherapy effects in aggressive non-Hodgkin's lymphoma. Acta Oncol 2001; 40: 198-212.

7. Goldie JH, Coldman AJ. The genetic origin of drug resistance in neoplasms: implications for systemic therapy. Cancer Res 1984; 44: 3643-3653.

8. Fisher RI, Gaynor ER, Dahlberg S, Oken MM, Grogan TM, Mize EM, et al. Comparison of a standard regimen (CHOP) with three intensive chemotherapy regimens for advanced non-Hodgkin's lymphoma. N Engl J Med 1993; 328: 1002-1006.

9. Philip T, Guglielmi C, Hagenbeek A, Somers R, Van Der LH, Bron D, et al. Autologous bone marrow transplantation as compared with salvage chemotherapy in relapses of chemotherapy-sensitive nonHodgkin's lymphoma. N Engl J Med 1995; 333: 1540-1545.

10. Cheson BD, Horning SJ, Coiffier B, Shipp MA, Fisher RI, Connors $\mathrm{JM}$, et al. Report of an international workshop to standardize response criteria for non-Hodgkin's lymphomas. NCI Sponsored International Working Group. J Clin Oncol 1999; 17: 1244.

11. Carbone PP, Kaplan HS, Musshoff K, Smithers DW, Tubiana M. Report of the Committee on Hodgkin's Disease Staging Classification. Cancer Res 1971; 31: 1860-1861.

12. Magrath I. Chemotherapy in developing countries - is less better? Eur J Cancer 2003; 39: 1497-1500.

13. Fisher RI, DeVita VT Jr, Hubbard SM, Longo DL, Wesley R, Chabner $\mathrm{BA}$, et al. Diffuse aggressive lymphomas: increased survival after alternating flexible sequences of proMACE and MOPP chemotherapy. Ann Intern Med 1983; 98: 304-309.

14. Montserrat E, Garcia-Conde J, Vinolas N, Lopez-Guillermo A, Hernandez-Nieto L, Zubizarreta A, et al. CHOP vs. ProMACECytaBOM in the treatment of aggressive non-Hodgkin's lymphomas: long-term results of a multicenter randomized trial. (PETHEMA:
Spanish Cooperative Group for the Study of Hematological Malignancies Treatment, Spanish Society of Hematology). Eur J Haematol 1996; 57: 377-383.

15. Gordon LI, Andersen J, Colgan J, Glick J, Resnick GD, O'Connell M, et al. Advanced diffuse non-Hodgkin's lymphoma. Analysis of prognostic factors by the international index and by lactic dehydrogenase in an intergroup study. Cancer 1995; 75: 865-873.

16. Coltman CA, Dahlberg S, Jones SE. CHOP is curative in thirty\% of patients with large cell lymphoma: a twelve-year Southwest Oncology Group follow-up. In: Skarin AT (Editor), Advances in cancer chemotherapy. New York: Park Row; 1986. p 71-78.

17. Miller TP, Dahlberg S, Cassady JR, Adelstein DJ, Spier CM, Grogan TM, et al. Chemotherapy alone compared with chemotherapy plus radiotherapy for localized intermediate- and high-grade non-Hodgkin's lymphoma. N Engl J Med 1998; 339: 21-26.

18. Coiffier B, Lepage E, Briere J, Herbrecht R, Tilly H, Bouabdallah R, et al. CHOP chemotherapy plus rituximab compared with $\mathrm{CHOP}$ alone in elderly patients with diffuse large-B-cell lymphoma. $N$ Engl J Med 2002; 346: 235-242.

19. Feugier $P$, Van HA, Sebban $C$, Solal-Celigny $P$, Bouabdallah $R$, Ferme $\mathrm{C}$, et al. Long-term results of the R-CHOP study in the treatment of elderly patients with diffuse large B-cell lymphoma: a study by the Groupe d'Etude des Lymphomes de l'Adulte. J Clin Oncol 2005; 23: 4117-4126.

20. Cabanillas F, Hagemeister FB, McLaughlin P, Velasquez WS, Riggs $S$, Fuller $L$, et al. Results of MIME salvage regimen for recurrent or refractory lymphoma. J Clin Oncol 1987; 5: 407-412.

21. Wilson WH, Bryant G, Bates S, Fojo A, Wittes RE, Steinberg SM, et al. EPOCH chemotherapy: toxicity and efficacy in relapsed and refractory non-Hodgkin's lymphoma. J Clin Oncol 1993; 11: 15731582.

22. Velasques WS, McLaughlin P, Tucker S, Hagemeister FB, Swan F, Rodriguez MA, et al. ESHAP-An effective chemotherapy regimen in refractory and relapsed lymphoma: A 4-year follow-up study. J Clin Oncol 1992; 12: 1176.

23. Shipp MA, Abeloff MD, Antman KH, Carroll G, Hagenbeek A, Loeffler $\mathrm{M}$, et al. International Consensus Conference on High-Dose Therapy with Hematopoietic Stem Cell Transplantation in Aggressive Non-Hodgkin's Lymphomas: report of the jury. J Clin Oncol 1999; 17: 423-429.

24. Hamlin PA, Zelenetz AD, Kewalramani T, Qin J, Satagopan JM, Verbel D, et al. Age-adjusted International Prognostic Index predicts autologous stem cell transplantation outcome for patients with relapsed or primary refractory diffuse large B-cell lymphoma. Blood 2003; 102: 1989-1996. 